\title{
Experimental test of entropic noise-disturbance uncertainty relations for three-outcome qubit measurements
}

\author{
Stephan Sponar $\odot,{ }^{1, *}$ Armin Danner $\odot,{ }^{1}$ Vito Pecile $\odot,{ }^{1}$ Nico Einsidler $\odot,{ }^{1}$ Bülent Demirel, ${ }^{2}$ and Yuji Hasegawa $\odot{ }^{1,3, \dagger}$ \\ ${ }^{1}$ Atominstitut, TU Wien, Stadionallee 2, 1020 Vienna, Austria \\ ${ }^{2}$ Institute for Functional Matter and Quantum Technologies, University of Stuttgart, 70569 Stuttgart, Germany \\ ${ }^{3}$ Department of Applied Physics, Hokkaido University, Kita-ku, Sapporo 060-8628, Japan
}

(Received 24 June 2020; revised 25 March 2021; accepted 19 May 2021; published 4 June 2021)

\begin{abstract}
Information-theoretic uncertainty relations formulate the joint immeasurability of two noncommuting observables in terms of information entropies. The tradeoff of the accuracy in the outcome of two successive measurements manifests in entropic noise-disturbance uncertainty relations. Recent theoretical analysis predicts that projective measurements are not optimal, with respect to the noise-disturbance tradeoffs. Therefore, the results in our previous paper [Phys. Rev. Lett. 115, 030401 (2015)] are outperformed by general quantum measurements. Here, we experimentally test a tight information-theoretic measurement uncertainty relation for three-outcome positive-operator-valued measures, using neutron spin- $\frac{1}{2}$ qubits. The obtained results violate the lower bound for projective measurements as theoretically predicted.
\end{abstract}

DOI: 10.1103/PhysRevResearch.3.023175

\section{INTRODUCTION}

According to the rules of quantum mechanics, any single observable or even a set of compatible observables can be measured with arbitrary accuracy. However, classically unanticipated consequences appear when measuring noncommuting observables jointly, either simultaneously or successively. Heisenberg's seminal paper from 1927 [1] predicts on one hand a lower bound on the uncertainty of a joint measurement of incompatible observables. On the other hand, it also sets an upper bound on the accuracy with which the values of noncommuting observables can be simultaneously prepared. While in the past these two statements have often been mixed, they are now clearly distinguished as measurement uncertainty and preparation uncertainty relations, respectively.

While Heisenberg's paper only presented his idea heuristically, the first rigorously proven uncertainty relation for position $Q$ and momentum $P$ was provided by Kennard [2] as $\Delta(Q) \Delta(P) \geqslant \frac{\hbar}{2}$, in terms of standard deviations defined as $\Delta^{2}(A)=\left\langle\psi\left|A^{2}\right| \psi\right\rangle-\langle\psi|A| \psi\rangle^{2}$. Hence, the positionmomentum uncertainty relation in terms of standard deviations quantifies how precise, with respect to the observables of interest, a state can be prepared, rather than the ability to jointly measure them. In 1929, Robertson [3] extended Kennard's relation to arbitrary pairs of observables $A$ and $B$

\footnotetext{
*stephan.sponar@tuwien.ac.at

†yuji.hasegawa@tuwien.ac.at
}

Published by the American Physical Society under the terms of the Creative Commons Attribution 4.0 International license. Further distribution of this work must maintain attribution to the author(s) and the published article's title, journal citation, and DOI. as

$$
\Delta(A) \Delta(B) \geqslant \frac{1}{2}|\langle\psi|[A, B]| \psi\rangle|,
$$

with the commutator $[A, B]=A B-B A$.

It is widely accepted [4] (but nevertheless under discussion $[5,6])$ that the uncertainty relation as formulated by Robertson in terms of standard deviations $\Delta(A,|\psi\rangle) \Delta(B,|\psi\rangle) \geqslant$ $\frac{1}{2}|\langle\psi|[A, B]| \psi\rangle|$ lacks an irreducible or state-independent lower bound, meaning it can become zero for noncommuting observables. Furthermore, the standard deviation is not an optimal measure for all states. Consequently, Deutsch began to seek a theorem of linear algebra in the form $\mathcal{U}(A, B, \psi) \geqslant$ $\mathcal{B}(A, B)$ and suggested to use (Shannon) entropy as an appropriate measure. Note that Heisenberg's (and Kennard's) inequality $\Delta(Q) \Delta(P) \geqslant \frac{\hbar}{2}$ has that particular form, but its generalization, Eq. (1), does not. Uncertainty relations in terms of entropy were introduced to solve both problems. The first entropic preparation uncertainty relation was formulated by Hirschman [7] in 1957 for the position and momentum observables, which was later improved in 1975 by Beckner [8] and Bialynicki-Birula and Mycielski [9]. The extension to nondegenerate observables on a finite-dimensional Hilbert space was given by Deutsch in 1983 [4] as

$$
H(A)+H(B) \geqslant-2 \log _{2}\left(\frac{1+c}{2}\right),
$$

where $H$ denotes the Shannon entropy and incompatibility $c=\max _{i, j}\left|\left\langle a_{i} \mid b_{j}\right\rangle\right|$ is the maximum overlap between the eigenvectors $\left|a_{i}\right\rangle$ and $\left|b_{j}\right\rangle$ of observables $A$ and $B$, respectively. This relation was later improved by Maassen and Uffink [10], following a conjecture of Kraus [11], yielding the well-known entropic uncertainty relation

$$
H(A)+H(B) \geqslant-2 \log _{2} c .
$$


(a)

$$
\begin{gathered}
\left|a_{\text {in }}\right\rangle=| \pm a\rangle \\
\text { with probability } \\
p( \pm)=\frac{1}{2} \\
\mathbb{A}<+ \text { correlations }
\end{gathered}
$$

(b)

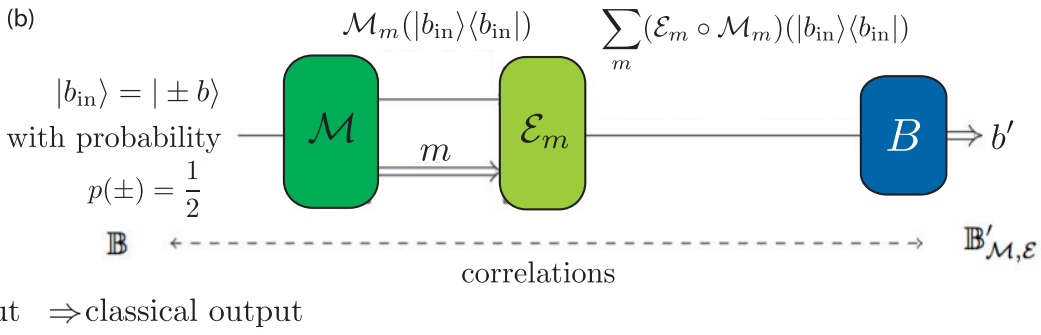

FIG. 1. Schematics of the scenarios used in the information-theoretic definitions of (a) noise $N(\mathcal{M}, A)$, and (b) disturbance $D_{\mathcal{E}}(\mathcal{M}, B)$ for two-level systems. The eigenstates $\left|a_{\text {in }}\right\rangle$ of $A$ (or $\left|b_{\text {in }}\right\rangle$ of $B$ for disturbance) are prepared with equal probability $p( \pm)=\frac{1}{2}$, before being measured by $\mathcal{M}$, producing outcome $m$ and transforming the state according to $\mathcal{M}_{m}$. (b) For the disturbance, the input states are $| \pm b\rangle$, again with probability $p( \pm)=\frac{1}{2}$. The result of the first measurement is classically communicated to a device applying a correction transformation $\mathcal{E}_{m}$ on the post-measurement state. The disturbance is obtained upon a subsequent projective measurement of $B$ yielding outcome $b^{\prime}$ at the end.

Entropic uncertainty has proven to be a useful tool in entanglement witnessing [12], complementarity [13], and in quantum information theory [14]. Initially, procedures to quantify error and disturbance are based on distance measures between target observables and measurements [5,15] or the associated probability distributions [16]. More recently, interest has risen in information-theoretic measures, introduced first by Buscemi et al. [17], but also in several subsequent alternative approaches [18-21].

\section{THEORY}

To formally study entropic measurement uncertainty relations one must define measures for two key properties of a measurement device, more precisely a quantum instrument $\mathcal{M}$ [22,23] (which may in general implement an arbitrary quantum measurement with any number of outcomes): how accurately it measures a target observable $A$ (noise), and how much it disturbs subsequent measurements (disturbance).

While several definitions of noise have previously been studied theoretically and experimentally, we utilize the information-theoretic approach of [17] (schematically illustrated in Fig. 1 in case of two-level systems) formulated as follows. Let $\{|a\rangle\}_{a}$ be the $a$ th eigenstates of the $d$ dimensional target observable $A$ and measurement device $\mathcal{M}$ being a collection $\left\{\mathcal{M}_{m}\right\}_{m}$ of completely positive (CP) tracenonincreasing maps $\mathcal{M}_{m}$. The instrument $\mathcal{M}$ uniquely defines a positive-operator-valued measure (POVM), denoted as $M=\left\{M_{m}\right\}_{m}$ [14]. The noise is defined in the following scenario: the eigenstates of $A$ are randomly prepared with probability $p(a)=\frac{1}{d}$ before $\mathcal{M}$ is applied, producing an outcome $m$ with probability $p(m \mid a)=\operatorname{Tr}\left(M_{m}|a\rangle\langle a|\right)$. If $\mathcal{M}$ accurately measures $A$, then the value of $m$ should allow one to infer $a$; if the measurement is noisy, $m$ yields less information about $a$. This noise is quantified in terms of the conditional Shannon entropy: denoting the random variables associated with $a$ and $m$ as $\mathbb{A}$ and $\mathbb{M}$, respectively, the noise of $\mathcal{M}$ for a measurement of $A$ is [17]

$$
N(\mathcal{M}, A)=H(\mathbb{A} \mid \mathbb{M})=-\sum_{a, m} p(a, m) \log _{2} p(a \mid m),
$$

where $p(a, m)=p(a) p(m \mid a)$ and $p(a \mid m)$ can be calculated from Bayes' theorem.

The entropic disturbance $D(\mathcal{M}, B)$ of the apparatus $\mathcal{M}$ on the measurement of $B$ is defined with respect to an analogous procedure as the noise. Uniformly distributed eigenstates $\left\{\left|b_{i}\right\rangle\right\}$ with eigenvalues $b_{i}$ associated with random variable $\mathbb{B}$ are fed to the same instrument $\mathcal{M}$ from which a post-measurement state $\rho_{m}=\frac{\mathcal{M}_{m}\left(\left|b_{i}\right\rangle\left\langle b_{i}\right|\right)}{\operatorname{Tr}\left(\mathcal{M}_{m}\left(\left|b_{i}\right\rangle\left\langle b_{i}\right|\right)\right)}$ emerges. In the disturbance configuration there is an additional subsequent measurement of observable $B$ with outcomes $\left\{b_{j}^{\prime}\right\}$. Due to the disturbing nature of the measurement apparatus $\mathcal{M}$, generally, a loss of correlation occurs. A subtle, yet important addendum to the concept of disturbance are error corrections. After measurement by $\mathcal{M}_{m_{j}}$, the state decomposed to the eigenstates of the measurement observables can be further transformed by a quantum operation $\mathcal{E}_{m}$ dependent on the pointer value $m$ of the apparatus. The disturbance $D_{\mathcal{E}}(\mathcal{M}, B)$ is defined as the conditional entropy $H\left(\mathbb{B} \mid \mathbb{B}_{\mathcal{M}, \mathcal{E}}^{\prime}\right)$ as

$D_{\mathcal{E}}(\mathcal{M}, B):=H\left(\mathbb{B} \mid \mathbb{B}_{\mathcal{M}, \mathcal{E}}^{\prime}\right)=-\sum_{i, j} p\left(b_{i}, b_{j}^{\prime}\right) \log _{2}\left(p\left(b_{i} \mid b_{j}^{\prime}\right)\right)$.

Using these notions of noise and disturbance, for arbitrary observables $A$ and $B$ in finite-dimensional Hilbert spaces, the noise-disturbance (measurement) relation

$$
N(\mathcal{M}, A)+D_{\mathcal{E}}(\mathcal{M}, B) \geqslant-\log _{2}\left(\max _{i, j}\left|\left\langle a_{i} \mid b_{j}\right\rangle\right|^{2}\right)
$$

holds [17]. In [24] we experimentally tested

$$
g[N(\mathcal{M}, A)]^{2}+g\left[D_{\mathcal{E}}(\mathcal{M}, B)\right]^{2} \leqslant 1,
$$

where $g[x]$ is the inverse of the function $h(x)$ defined as

$$
h(x)=-\frac{1+x}{2} \log _{2}\left(\frac{1+x}{2}\right)-\frac{1-x}{2} \log _{2}\left(\frac{1-x}{2}\right),
$$

with $x \in[0,1]$, for maximally incompatible qubit observables $A$ and $B$. As it turned out, the proof given in [24] for this relation was incorrect and this relation does not hold in general, which was pointed out in [25]. It should be noted that the relation does hold for projective measurements $\mathcal{M}_{\mathrm{pr}}$, although it can be violated by nonprojective measurements.

The bound of Eq. (7) can be violated by considering a three-outcome measurement $\mathcal{M}^{\theta}$, proposed in [25], with the associated positive-operator-valued measure (POVM) given by $M^{\theta}=\left\{M_{+1}^{\theta}, M_{0}^{\theta}, M_{-1}^{\theta}\right\}$ for $\theta \in\left[0, \frac{\pi}{2}\right]$, where

$$
\begin{aligned}
M_{m}^{\theta} & =p_{m}\left[\mathbb{1}+\boldsymbol{n}(\theta)_{m} \cdot \boldsymbol{\sigma}\right] \quad \text { with weights and directions, } \\
p_{0} & =\frac{\cos \theta}{1+\cos \theta}, \quad p_{-1}=p_{1}=\frac{1}{2(1+\cos \theta)} \\
\boldsymbol{n}(\theta)_{m} & =\left((-1)^{m} \cos (m \theta), 0, \sin (m \theta)\right)^{T} .
\end{aligned}
$$



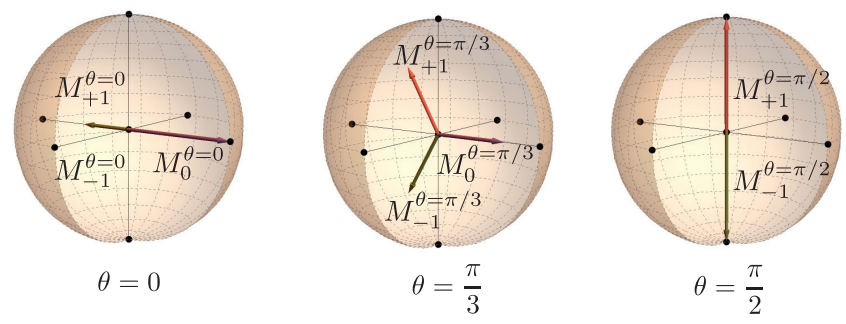

FIG. 2. Bloch sphere representation of the three-outcome POVM $M^{\theta}=\left\{M_{-1}^{\theta}, M_{0}^{\theta}, M_{+1}^{\theta}\right\}$ for three selected values of the parameter $\theta$, given by $\theta=0, \pi / 3, \pi / 2$.

In Fig. 2 the POVM $M_{m}^{\theta}$ is illustrated for three distinctive values of the parameter $\theta$. The POVM $M_{m}^{\theta}$ is conjectured to be optimal, while a rigorous proof is still pending. However, the numerical simulations presented in [25] suggest that the POVM $M_{m}^{\theta}$ is optimal. Note that for $\theta=\frac{\pi}{2}$ the POVM $M^{\theta}$ degenerates to a projective measurement in $\pm z$ direction with elements $M_{-1}^{\theta=\pi / 2}=|-z\rangle\langle-z|$ and $M_{+1}^{\theta=\pi / 2}=|+z\rangle\langle+z|$, a projective measurement of $A=\sigma_{z}$ resulting in zero noise. While for $\theta=0$ the POVM element $M_{0}^{\theta=0}$ represents the projector $|+x\rangle\langle+x|$, de facto accounting for a projective measurement of $B=\sigma_{x}$, therefore zero disturbance is expected.

\section{Noise and disturbance for the three-outcome POVM $M^{\theta}$}

The probability of obtaining outcome $m$ when measuring a state $\rho$ is thus $\operatorname{Tr}\left[\mathrm{M}_{\mathrm{m}} \rho\right]$. Plugging in the three-outcome POVM $M^{\theta}$ from Eq. (9) into the definition of noise

$$
\begin{aligned}
N\left(\mathcal{M}^{\theta}, A\right) & =H(\mathbb{A} \mid \mathbb{M})=-\sum_{a, m} p(a, m) \log _{2} p(a \mid m) \\
& =-\sum_{m} p(m) \sum_{a} p(a \mid m) \log _{2} p(a \mid m),
\end{aligned}
$$

we calculate the noise on $A=\sigma_{z}$. The theoretical predictions for the conditional probabilities $p(a \mid m)$ are given by

$$
\begin{aligned}
p(a \mid m) & =\operatorname{Tr}\left[|a\rangle\langle a| \frac{M_{m}}{\operatorname{Tr}\left[M_{m}\right]}\right] \\
& =\frac{1}{2}(1+m a \sin \theta)|m|+(1-|m|) \cos \theta .
\end{aligned}
$$

With the six conditional probabilities $p(a \mid m)$ we can calculate the noise $N\left(\mathcal{M}^{\theta}, \sigma_{z}\right)$ via Eq. (10) with $p(m)=\frac{1}{d} \operatorname{Tr}\left[M_{m}^{\theta}\right]=$ $\frac{1}{2} \operatorname{Tr}\left[M_{m}^{\theta}\right]$ (since for qubits we have $d=2$ ) as

$$
N\left(\mathcal{M}^{\theta}, \sigma_{z}\right)=\frac{\cos \theta+h(\sin \theta)}{1+\cos \theta},
$$

with $h(x)$ defined as

$$
h(x)=-\frac{1+x}{2} \log _{2}\left(\frac{1+x}{2}\right)-\frac{1-x}{2} \log _{2}\left(\frac{1-x}{2}\right)
$$

with $x \in[0,1]$.

In order to determine a lower bound on the disturbance $D\left(\mathcal{M}^{\theta}, \sigma_{x}\right)$, let us consider the optimal correction $\mathcal{E}_{m}^{\text {opt }}$ that maps $\boldsymbol{n}_{-1}$ and $\boldsymbol{n}_{1}$ onto the negative $x$ axis and $\boldsymbol{n}_{0}$ onto the positive $x$ axis, respectively, denoted as

$$
\mathcal{E}_{m}^{\mathrm{opt}}\left(\boldsymbol{n}(\theta)_{m}\right)=\frac{11+(-1)^{m} \boldsymbol{e}_{x} \cdot \boldsymbol{\sigma}}{2} .
$$

Using

$$
\begin{aligned}
D_{\mathcal{E}}\left(\mathcal{M}^{\theta}, B\right): & =H\left(\mathbb{B} \mid \mathbb{B}_{\mathcal{M}^{\theta}, \mathcal{E}}^{\prime}\right)=-\sum_{b, b^{\prime}} p\left(b, b^{\prime}\right) \log \left[p\left(b \mid b^{\prime}\right)\right] \\
& =-\sum_{b, b^{\prime}} p\left(b, b^{\prime}\right) \log _{2} \frac{p\left(b, b^{\prime}\right)}{p\left(b^{\prime}\right)}
\end{aligned}
$$

we get for the joint probabilities

$$
\begin{aligned}
p\left(b, b^{\prime}\right) & =\sum_{m=-1}^{1} p_{m}\left(\frac{1+b \boldsymbol{e}_{x} \cdot \boldsymbol{n}(\theta)_{m}}{2}\right)\left(\frac{1+(-1)^{m} b^{\prime}}{2}\right) \\
& =\frac{1-b^{\prime}+\left(1+b^{\prime}+2 b b^{\prime}\right) \cos \theta}{4(1+\cos \theta)} .
\end{aligned}
$$

Marginal probabilities are given by summation

$$
p\left(b^{\prime}\right)=\sum_{b} p\left(b, b^{\prime}\right)=\frac{1-b^{\prime}+\cos \theta+b^{\prime} \cos \theta}{2+2 \cos \theta} .
$$

Finally, the disturbance is calculated applying the four joint probabilities $p\left(b, b^{\prime}\right)$ from above via the conditional entropy $H\left(\mathbb{B} \mid \mathbb{B}^{\prime}\right)$, from Eq. (26), with $B=\sigma_{x}$ as

$$
D_{\mathcal{E}}\left(\mathcal{M}^{\theta}, \sigma_{x}\right)=\frac{h(\cos \theta)}{1+\cos \theta} .
$$

This noise-disturbance pair from Eqs. (12) and (18) violates Eq. (7) for all $\theta \in] 0, \frac{\pi}{2}[$, which is experimentally tested here.

\section{EXPERIMENT}

\section{A. Experimental setup}

The experiment was performed at the polarimeter instrument NepTUn (NEutron Polarimeter TU wieN) [26-31], located at the tangential beam port of the 250-kW TRIGA Mark II research reactor at the Atominstitut-TU Wien, in Vienna, Austria. A schematic illustration of the experimental setup is depicted in Fig. 3. As in our previous experiments [24] the qubit system studied in the present experiment is represented by the spin, a two-state system, of the spin- $\frac{1}{2}$ particle neutron, where $\mathcal{S}_{i}=\frac{\hbar}{2} \sigma_{i}$, with $i=x, y, z$. An incoming monochromatic neutron beam with mean wavelength $\lambda \simeq$ $2.02 \AA(\Delta \lambda / \lambda \simeq 0.02)$ is polarized along the vertical $(+z)$ direction by refraction from a CoTi multilayer array, henceforth referred to as supermirror. To prevent depolarization by stray fields, a 13 Gauss guide field $B_{z}^{\mathrm{GF}}$ pointing in the positive $z$ direction, from coils in Helmholtz configuration, is applied along the entire setup (Helmholtz coils not depicted in Fig. 3). The probability of preparation of one of the two possible initial spin states, that is $\left|S_{z} ; \pm\right\rangle:=| \pm z\rangle$ for noise and $\left|S_{x} ; \pm\right\rangle:=| \pm x\rangle$ for disturbance measurement, is determined by a classical random-number generator applying one out of two possible currents in the spin rotator coil DC-1. Within the coil DC-1 a local magnetic field $B_{y}$, pointing in positive $y$ direction, is applied. Larmor precession around the $y$ axis is induced and the strength of $B_{y}$ is tuned such that it causes a 


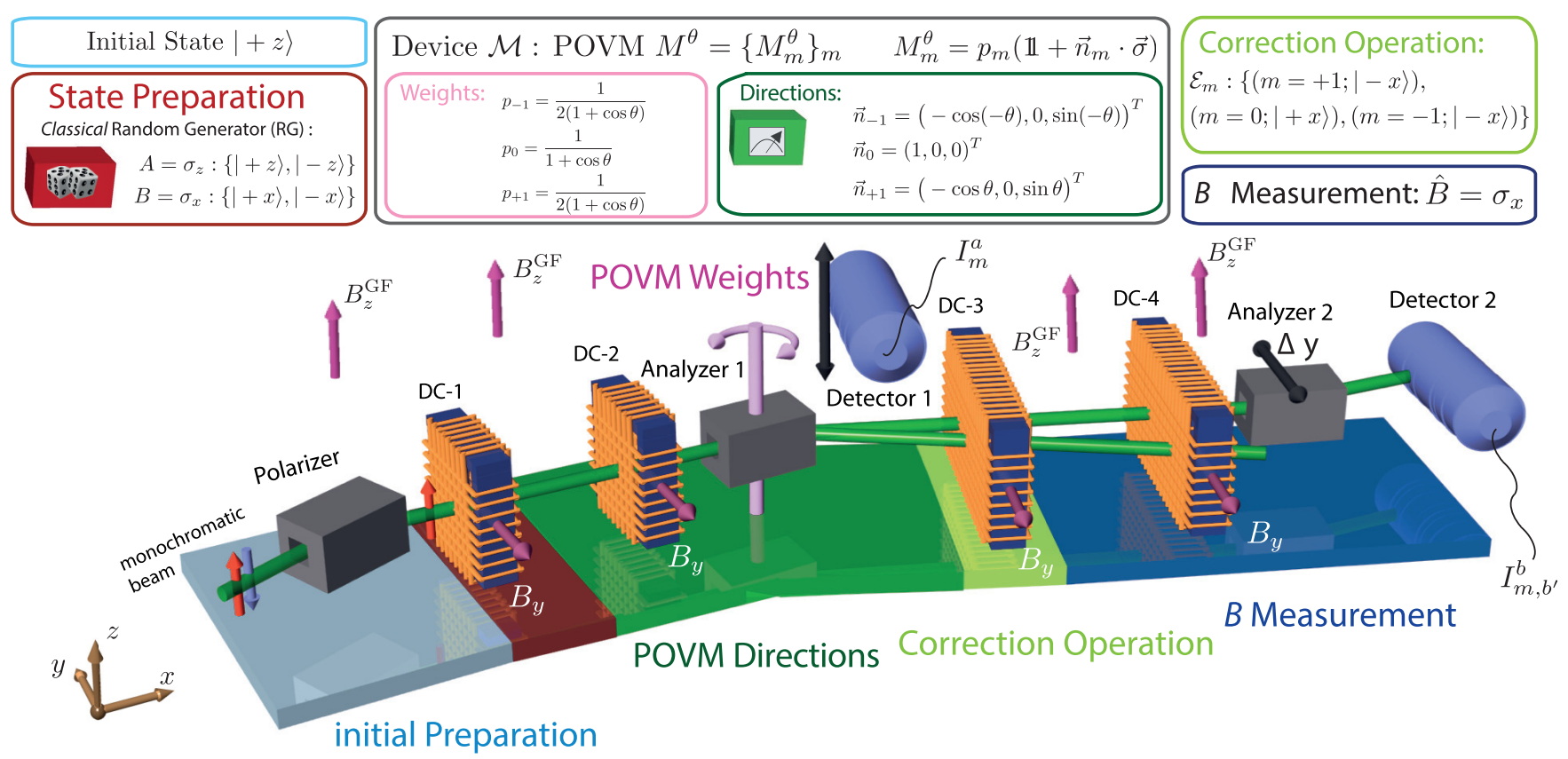

FIG. 3. Schematic illustration of the neutron polarimetric setup for noise-disturbance measurement of $\mathcal{M}$, representing a complete quantum instrument, consisting of three-outcome POVM $M^{\theta}$, transformation of post-measurement state (correction operation), and projective measurement $B$. The illustration includes a descriptive legend of the different experimental regions. The setup consists of three supermirror arrays (one polarizer, two analyzers), four direct current coils (DC-1, 2, 3, 4), and two detectors. Exploiting Larmor precession of the Bloch vector around magnetic fields $\left(B_{y}, B_{z}^{\mathrm{GF}}\right)$ and using supermirror arrays to realize direction of projectors and weights, all required spin states are prepared and the three-outcome POVM $M^{\theta}$, as well as a projective measurement $\sigma_{x}$, is implemented. Noise $N\left(\mathcal{M}, \sigma_{z}\right)$ and disturbance $D_{\mathcal{E}}\left(\mathcal{M}, \sigma_{x}\right)$ are evaluated from the measured intensities $I_{m}^{a}$ and $I_{m, b^{\prime}}^{b}$, respectively.

spin rotation by an angle of 0 or $\pi$ for the noise and $+\frac{\pi}{2}$ or $-\frac{\pi}{2}$ for the disturbance measurement, respectively.

For the three-outcome POVM $M^{\theta}$ another spin rotator coil (DC-2) and the second supermirror (analyzer 1) are applied. As seen from the definition of the POVM $M_{m}^{\theta}=p_{m}(\theta)[\mathbb{1}+$ $\boldsymbol{n}(\theta)_{m} \cdot \boldsymbol{\sigma}$ ], each POVM element consists of a measurement direction given by $\boldsymbol{n}_{m}$ and a weighting denoted as $p_{m}$, dependent on the parameter $\theta$. While the former is adjusted by an appropriate magnetic field strength $B_{y}$ in DC-2, the latter is set by the horizontal angle of refraction inside the supermirror. Note that the change in angle of the supermirror only effects the transmission (weighting) and does not change polarization of the neutrons, making this procedure a valid experimental realization of the POVM $M^{\theta}$.

For the noise-disturbance measurement the whole function of the quantum instrument has to be specified (not just the POVM it induces), which includes transformation of the postmeasurement state. Consequently, a correction operation $\mathcal{E}_{m}^{\text {opt }}$ is applied, in order to minimize the disturbance $D_{\mathcal{E}}\left(\mathcal{M}^{\theta}, B\right)$. In our experiment $\mathcal{E}_{m}^{\text {opt }}$ maps $\boldsymbol{n}_{ \pm 1}$ onto the negative $x$ axis and $\boldsymbol{n}_{0}$ onto the positive $x$ axis, which is achieved by Larmor precession with DC-3. Finally, DC-4 and the third supermirror (second analyzer) perform the $B$ measurement, which is a simple projective measurement, where the observable is given by $B=\sigma_{x}$. At the end of the beam line a boron trifluoride counting tube (detector 2 in Fig. 3) registers all incoming neutrons. The two successively performed measurements of $M^{\theta}$ and $B$ result in six output intensities $I_{m, b^{\prime}}^{b}$ for $B=\sigma_{x}$ (disturbance measurement), for each setting of $\theta$. For the noise measurement no $B$ measurement is required, thus, only three output intensities $I_{m}^{a}$ (with $\left.m=-1,0,1\right)$ are obtained, in an additionally inserted counting tube (detector 1 in Fig. 3).

\section{B. Noise and disturbance measurements}

Uniformly distributed eigenstates of the observable $A=$ $\sigma_{z}$, denoted as $\left\{\left|a_{i}\right\rangle\right\}=\{|+z\rangle,|-z\rangle\}$, are sent onto the apparatus $\mathcal{M}^{\theta}$. The correlation between the eigenvalue $a_{i}$ corresponding to the state prepared and the outcome $m$ measured by the apparatus $\mathcal{M}$ is used to determine the noise $N\left(\mathcal{M}^{\theta}, A\right)$. This correlation is quantitatively characterized by the joint probability distribution $p(a, m)$. The conditional probability $p(a \mid m)$ is then obtained via $p(a \mid m)=\frac{p(a, m)}{p(m)}$, allowing to calculate the noise $N\left(\mathcal{M}^{\theta}, A\right)$ using Eq. (10). The noise $N\left(\mathcal{M}^{\theta}, A\right)$ of the three-outcome POVM $M^{\theta}$ is determined applying the reduced setup, where the detector $\left({ }^{3} \mathrm{He}\right.$ cylindric count tube, diameter $\emptyset=1$ in) is directly mounted onto the exit window of the second supermirror to maintain optimal positioning when the supermirror is rotated (to implement the POVM weights). With this configuration, a maximal count rate $I_{\max }=350$ counts per second is recorded. During the measurement the POVM parameter $\theta$ is varied between $\pi / 2$ and 0 in steps of $\pi / 34$. For each value of $\theta$ three intensities, belonging to the POVM outputs $M_{0}^{\theta}, M_{1}^{\theta}$, and $M_{-1}^{\theta}$ (denoted as $I_{m}^{a}$ with $m=-1,0,1$ ) are recorded in a measurement time $t_{\text {meas }}=400 \mathrm{~s}$, which is plotted in Fig. 4(a). The particular order of the POVM elements, that is starting with $M_{0}^{\theta}$ followed by $M_{+1}^{\theta}$ and $M_{-1}^{\theta}$, has experimental reasons, namely, to reduce the number of movements of the neutron optical components. For each value of $\theta$ an initial 


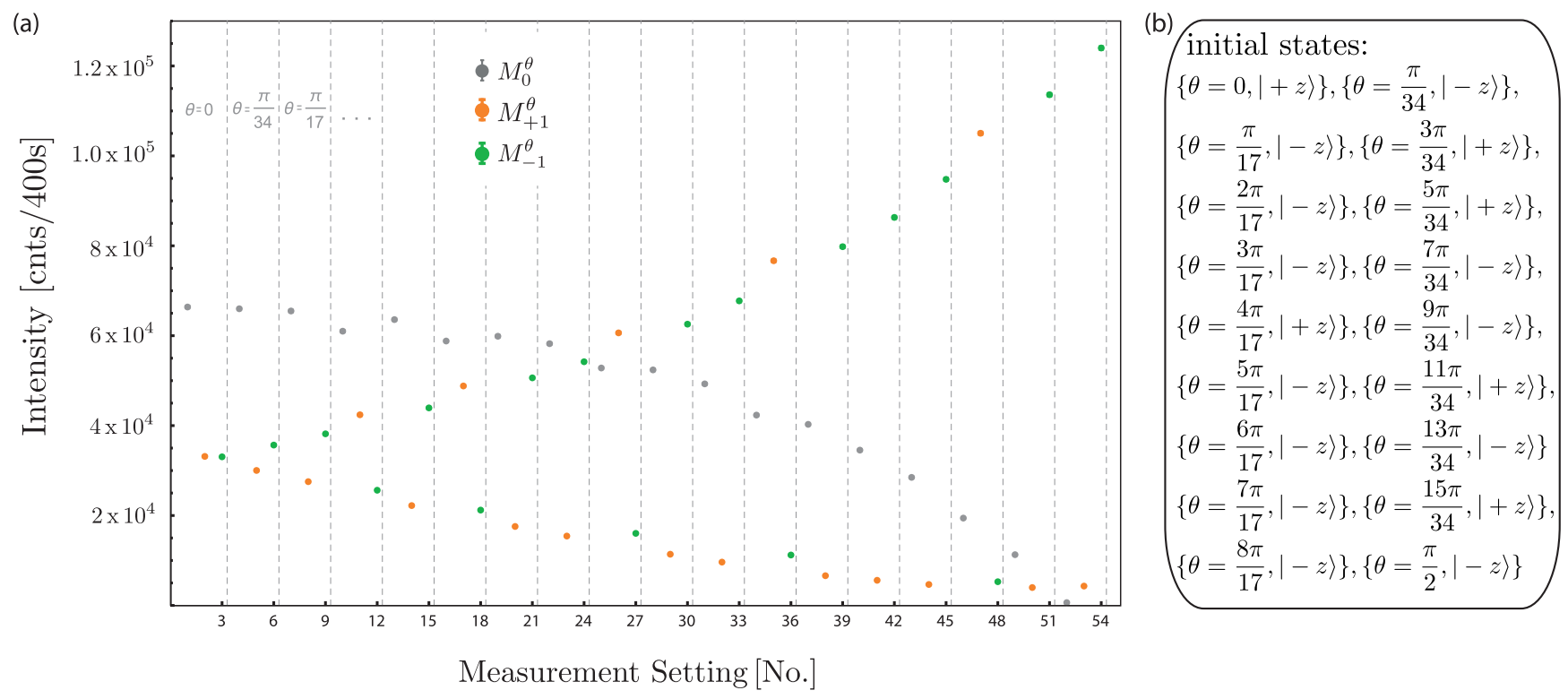

FIG. 4. (a) Raw data $I_{m}^{a}$ (with $\left.m=-1,0,+1\right)$ of the noise measurement $N\left(M^{\theta}, \sigma_{z}\right)$ of the three-outcome POVM $M^{\theta}$ for a measurement time of $400 \mathrm{~s}$. Error bars ( \pm 1 standard deviation) are below the size of points. (b) Randomly selected initial states $\left\{\left|a_{i}\right\rangle\right\}=\{|+z\rangle,|-z\rangle\}$ for all values of $\theta$.

state (eigenstate of $A=\sigma_{z}$ ) is chosen by random generator. The result is blinded during the measurement but stored in a data file for a later comparison with the obtained values for the noise $N(\mathcal{M}, A)$. The randomly generated sequence is listed in Fig. 4(b).

The count rates are detangled according to their corresponding POVM output, and data corrections are performed: First a background correction is applied, by subtraction the background counts of $I_{m}^{\mathrm{bg}}=1.37 \pm 0.03$ counts per second resulting in the intensity ${ }^{\text {bgCorr }} I_{m}^{a}$. A second correction is performed, by taking the finite contrast for our system, measured as $C=95 \%$ into account.

Next, the count rates are normalized by the total count rate. The statistical error is given by square root of the observed count rate $\sqrt{N}$ (due to Poissonian statistics of the neutron count rates), before calculating the normalized count rate. The systematic error stems from the imperfection of the spin rotators and is estimated as $\sim 0.7^{\circ}$. Normalized data of $M_{+1}^{\theta}, M_{0}^{\theta}$, and $M_{-1}^{\theta}$, accounting for the joint probability

$$
p(a, m)=\frac{\operatorname{bgCorr}^{a} I_{m}^{a}}{\sum_{m}^{\text {bgCorr }} I_{m}^{a}},
$$

is plotted in Fig. 5. Using the theoretical prediction of the sum of the two probabilities given by $p(a= \pm 1, m=+1)=$ $p(a=+1, m=+1)+p(a=-1, m=+1)$, the joint probabilities $p(a=+1, m=+1)$ and $p(a=-1, m=+1)$ can be derived for each individual value of $\theta$.

The results for $M_{+1}^{\theta}$ are plotted in Fig. 5(a); apart from $\theta=0$, where the initial states are indistinguishable, the initial state can be inferred with a distinctive probability from $p(a=+1 \vee a=-1, m=+1)$. For the next output element that is $M_{0}^{\theta}$ the situation is different. As can be seen from the normalized count rate of $M_{0}^{\theta}$, which is plotted below in Fig. 5(b), it is impossible to infer which eigenstate of $\sigma_{z}$ was sent since the theoretical predictions are exactly the same.
Finally, we take a look at the third output element, that is $M_{-1}^{\theta}$, which is depicted in Fig. 5(c). Note that all theory curves from the output port $M_{-1}^{\theta}$ for input state $|+z\rangle$ correspond to those of $M_{+1}^{\theta}$ for input state $|-z\rangle$. Using

$$
p(a \mid m)=\frac{p(a, m)}{p(m)}=\frac{p(a, m)}{\sum_{a} p(a, m)},
$$

the conditional probabilities $p(a=+1 \mid m=+1)$ and $p(a=$ $-1 \mid m=+1)$ are calculated, which is depicted together with the theoretical predictions in Fig. 5(d). The identical data sets of $M_{0}^{\theta}$ are taken for the joint probabilities $p(a=+1, m=$ $0)=p(a=-1, m=0)$ and for the conditional probabilities $p(a=+1 \mid m=0)=p(a=-1 \mid m=0)$, which is plotted in Fig. 5(e). The conditional probabilities $p( \pm a \mid m=-1)$ are determined in analogous manner from $p(a=+1 \vee-1, m=$ $-1)$ via $p(a=+1, m=-1)$ and $p(a=-1, m=-1)$ resulting in $p(a=+1 \mid m=-1)$ and $p(a=-1 \mid m=-1)$, which is illustrated in Fig. 5(f). The theoretical predictions (red and blue curves in Fig. 5) for the conditional probabilities $p(a \mid m)$ are given by

$$
p(a \mid m)=\frac{1}{2}(1+m a \sin \theta)|m|+(1-|m|) \cos \theta .
$$

With the six conditional probabilities $p(a \mid m)$ we can calculate the noise $N\left(\mathcal{M}^{\theta}, \sigma_{z}\right)$ via

$$
N\left(\mathcal{M}^{\theta}, \sigma_{z}\right)=-\sum_{m} p(m) \sum_{a} p(a \mid m) \log _{2} p(a \mid m),
$$

with $p(m)=\frac{1}{2} \operatorname{Tr}\left[M_{m}^{\theta}\right]$. The final results of the noise measurement $N\left(\mathcal{M}^{\theta}, \sigma_{z}\right)$, together with the theoretic prediction

$$
N\left(\mathcal{M}^{\theta}, \sigma_{z}\right)=\frac{\cos \theta+h(\sin \theta)}{1+\cos \theta}
$$

for the three-outcome POVM measurement and

$$
N\left(\mathcal{M}_{\mathrm{pr}}, \sigma_{z}\right)=h(\cos \theta),
$$



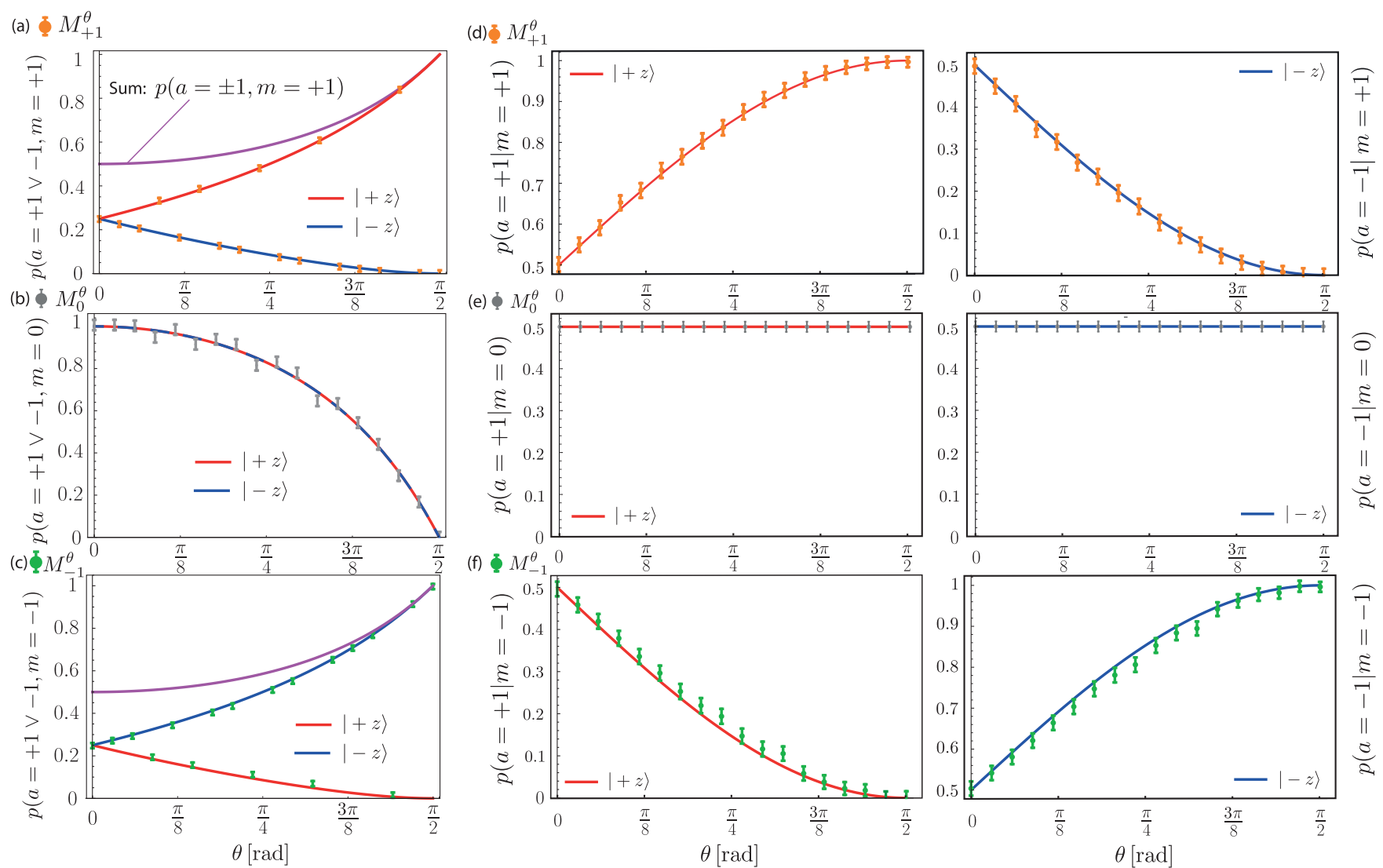

FIG. 5. Normalized data from Eq. (19), of $M_{+1}^{\theta}, M_{0}^{\theta}$, and $M_{-1}^{\theta}$ in (a)-(c), respectively, with "indefinite" input state (|+z〉 or $\left.|-z\rangle\right)$. Conditional probability $p(a= \pm 1 \mid m=-1,0,+1)$, by applying Eq. (20), for $|+z\rangle$ branch left and $|-z\rangle$ right plotted in (d)-(f).

for projective measurements, can be seen in Fig. 6 (blue and green curve, respectively).

For the disturbance measurement $D_{\mathcal{E}}\left(\mathcal{M}^{\theta}, B\right)$ the threeoutcome POVM measurement is followed by a subsequent projective measurement of an observable $B=\sigma_{x}$. In addition, an optimal correction operation $\mathcal{E}_{m}^{\text {opt }}$ in-between the two mea-

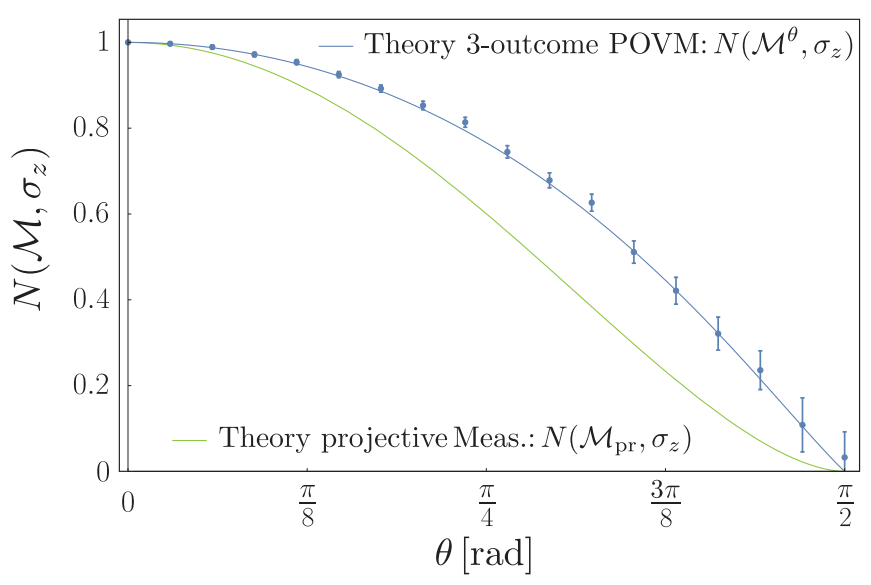

FIG. 6. Plot of the noise $N\left(\mathcal{M}^{\theta}, \sigma_{z}\right)$ of the three-outcome POVM $M^{\theta}$ as a function of the POVM parameter $\theta$, together with the theoretical predictions for POVM and projective measurements. Error bars correspond to plus or minus one standard deviation. surements maps $\boldsymbol{n}_{-1}$ and $\boldsymbol{n}_{1}$ onto the negative $x$ axis and $\boldsymbol{n}_{0}$ onto the positive $x$ axis, respectively.

Uniformly distributed eigenstates of the observable $B$, denoted as $\left\{\left|b_{i}\right\rangle\right\}=\{|+x\rangle,|-x\rangle\}$ and associated with random variable $\mathbb{B}$, are fed to the same instrument $\mathcal{M}^{\theta}$. Due to the disturbing nature of the measurement apparatus $\mathcal{M}^{\theta}$, generally, a loss of correlation occurs. The correlation between the eigenvalue $b$ corresponding to the state prepared and the outcome $b^{\prime}$ of the second now projective measured, which will be used to define the disturbance, is characterized by the joint probability distribution $p\left(b, b^{\prime}\right)$, allowing to calculate the disturbance $D_{\mathcal{E}}\left(\mathcal{M}^{\theta}, B\right)$ using Eq. (15).

In the actual experiment, the detector (boron trifluoride cylindric count tube, diameter $\varnothing=3$ in, active volume of length $L_{\text {act }}=30 \mathrm{~cm}$ ) was placed horizontally, transversal to the beam. This was done to account for the beam displacement $\Delta y \sim 10 \mathrm{~mm}$, caused by the tilt of the second supermirror, when setting the POVM weights. With this configuration a maximal count rate $I_{\max }=25 \mathrm{cnts} / \mathrm{s}$ is recorded. During the measurement the POVM parameter $\theta$ is varied between $\pi / 2$ and 0 in steps of $\pi / 34$. For each value of $\theta$ now six intensities $I_{m, b^{\prime}}^{b}$, belonging to the $+b$ and $-b$ measurements of the POVM outputs $M_{0}^{\theta}, M_{+1}^{\theta}$, and $M_{-1}^{\theta}$, are recorded in a measurement time $t_{\text {meas }}=400 \mathrm{~s}$, which is plotted in Fig. 7(a) (for higher statistics also a second data set with $t_{\text {meas }}=800 \mathrm{~s}$ was recorded). For each value of $\theta$ an initial state (eigenstate of $B=\sigma_{x}$ ) is chosen by a random generator. Again, the result is blinded during the measurement but stored in a data file for 


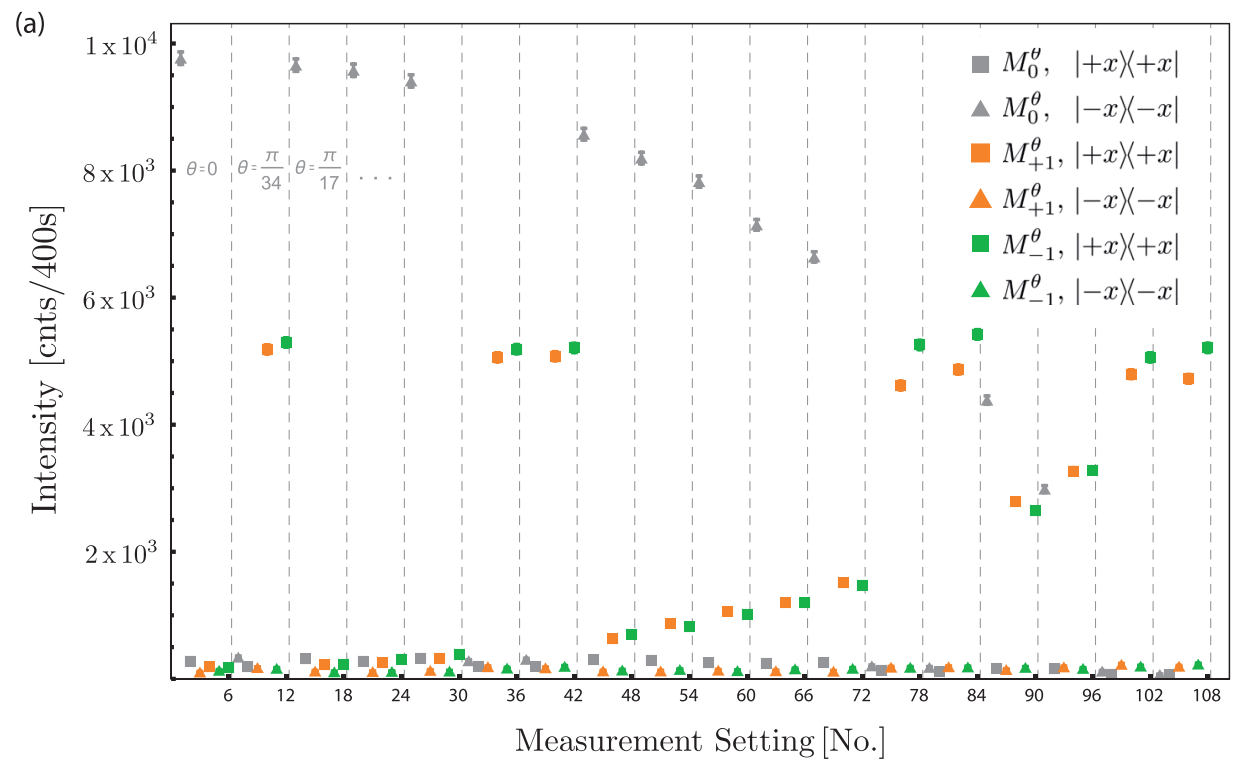

(b) $\left.\begin{array}{l}\text { initial states: } \\ \{\theta=0,|+x\rangle\},\left\{\theta=\frac{\pi}{34},|-x\rangle\right\}, \\ \left\{\theta=\frac{\pi}{17},|+x\rangle\right\},\left\{\theta=\frac{3 \pi}{34},|+x\rangle\right\}, \\ \left\{\theta=\frac{2 \pi}{17},|+x\rangle\right\},\left\{\theta=\frac{5 \pi}{34},|-x\rangle\right\}, \\ \left\{\theta=\frac{3 \pi}{17},|-x\rangle\right\}\left\{\theta=\frac{7 \pi}{34},|+x\rangle\right\}, \\ \left\{\theta=\frac{4 \pi}{17},|+x\rangle\right\},\left\{\theta=\frac{9 \pi}{34},|+x\rangle\right\}, \\ \left\{\theta=\frac{5 \pi}{17},|+x\rangle\right\},\left\{\theta=\frac{11 \pi}{34},|+x\rangle\right\}, \\ \left\{\theta=\frac{6 \pi}{17},|-x\rangle\right\},\left\{\theta=\frac{13 \pi}{34},|-x\rangle\right\} \\ \left\{\theta=\frac{7 \pi}{17},|+x\rangle\right\},\left\{\theta=\frac{15 \pi}{34},|+x\rangle\right\}, \\ \left\{\theta=\frac{8 \pi}{17},|-x\rangle\right\},\left\{\theta=\frac{\pi}{2},|-x\rangle\right\}\end{array}\right\}$

FIG. 7. (a) Raw data $I_{m \cdot b^{\prime}}^{b}$ (with $m=-1,0,+1$ and $b^{\prime}= \pm 1$ ) of the disturbance measurement $D_{\mathcal{E}}\left(M^{\theta}, \sigma_{x}\right)$ of the three-outcome POVM $M^{\theta}$ and projective $B=\sigma_{x}$ measurement for 400 s. (b) Randomly selected initial states $\left\{\left|b_{i}\right\rangle\right\}=\{|+x\rangle,|-x\rangle\}$ for all values of $\theta$.

a later comparison with the obtained values for the disturbance $D_{\mathcal{E}}\left(\mathcal{M}^{\theta}, B\right)$. The randomly generated sequence is listed in Fig. 7(b).

As before in the noise measurement, the count rates are detangled according to their corresponding $B$ measurement and POVM output.

Next, a background correction is applied, by subtracting the background counts of $I_{\mathrm{bg}}^{b^{\prime}}=0.176 \pm 0.008 \mathrm{cnts}$ per s resulting in the intensity ${ }^{\operatorname{bgCorr}} I_{m, b^{\prime}}^{b}$ and an overall contrast of $C=0.97$ is taken into account. Following the same procedure as for the noise, the count rates are normalized (equipped with statistical and systematical error) by the total number of counts which gives the six probabilities $p(b=+1 \vee$ $\left.-1, m, b^{\prime}\right)$ with $m=-1,0,1$ and $b^{\prime}= \pm 1$, which is plotted in Figs. 8(a)-8(c), respectively, and calculated as

$$
p\left(m, b, b^{\prime}\right)=\frac{\operatorname{bgCorr}^{b} I_{m, b^{\prime}}^{b}}{\sum_{b, b^{\prime}}^{\mathrm{bgCorr}} I_{m, b^{\prime}}^{b}} .
$$

Again, the data points are separated according to the input state $|+x\rangle \rightarrow b=+1$ and $|-x\rangle \rightarrow b=-1$, which gives in total 12 probabilities $p\left(m, b, b^{\prime}\right)$ with $m=-1,0,1, b= \pm 1$, and $b^{\prime}= \pm 1$. Since the disturbance is defined as

$$
\begin{aligned}
D_{\mathcal{E}}\left(\mathcal{M}^{\theta}, B\right) & :=H\left(\mathbb{B} \mid \mathbb{B}_{\mathcal{M}^{\theta}, \mathcal{E}}^{\prime}\right)=-\sum_{b, b^{\prime}} p\left(b, b^{\prime}\right) \log _{2} p\left(b \mid b^{\prime}\right) \\
& =-\sum_{b, b^{\prime}} p\left(b, b^{\prime}\right) \log _{2} \frac{p\left(b, b^{\prime}\right)}{p\left(b^{\prime}\right)}
\end{aligned}
$$

we have to calculate the joint probability $p\left(b, b^{\prime}\right)$ via

$$
p\left(b, b^{\prime}\right)=\sum_{m=-1}^{1} p\left(m, b, b^{\prime}\right),
$$

which is plotted in Fig. 9(a). The theoretical curves are given by

$$
p\left(b, b^{\prime}\right)=\frac{1-b^{\prime}+\left(1+b^{\prime}+2 b b^{\prime}\right) \cos \theta}{4(1+\cos \theta)},
$$

plotted as purple lines in Fig. 9(a). Next, we calculate the marginal probabilities $p\left(b^{\prime}\right)$ by summation of the data from above

$$
p\left(b^{\prime}\right)=\sum_{b} p\left(b, b^{\prime}\right),
$$

which is plotted in Fig. 9(b). The theoretical curves are given by

$$
p\left(b^{\prime}\right)=\frac{1-b^{\prime}+\cos \theta+b^{\prime} \cos \theta}{2+2 \cos \theta},
$$

plotted as gray lines in Fig. 9(b). Finally, the disturbance is calculated applying the four joint probabilities $p\left(b, b^{\prime}\right)$ from above via the conditional entropy $H\left(\mathbb{B} \mid \mathbb{B}_{\mathcal{M}^{\theta}, \mathcal{E}}^{\prime}\right)$ from Eq. (26). The final results of the disturbance measurement $D_{\mathcal{E}}\left(\mathcal{M}^{\theta}, \sigma_{x}\right)$, together with the theoretic prediction

$$
D_{\mathcal{E}}\left(\mathcal{M}^{\theta}, \sigma_{x}\right)=\frac{h(\cos \theta)}{1+\cos \theta}
$$

for the three-outcome POVM measurement and

$$
D_{\mathcal{E}}\left(\mathcal{M}_{\mathrm{pr}}, \sigma_{x}\right)=h(\sin \theta)
$$

for projective measurements, can be seen in Fig. 10, where the disturbance $D_{\mathcal{E}}\left(\mathcal{M}^{\theta}, \sigma_{x}\right)$ is plotted versus the noise $N\left(\mathcal{M}^{\theta}, \sigma_{z}\right)$ in comparison with results from suboptimal projective measurements $N\left(\mathcal{M}_{\mathrm{pr}}, \sigma_{z}\right)$ versus $D_{\mathcal{E}}\left(\mathcal{M}_{\mathrm{pr}}, \sigma_{x}\right)$.

The experimental results of the disturbance measurement $D_{\mathcal{E}}\left(\mathcal{M}^{\theta}, \sigma_{x}\right)$ can be seen in Fig. 10. The values obtained for the disturbance measurement for small values of $\theta$ are slightly higher than the theoretically predicted. This is due to the fact that for small values of $p\left(b, b^{\prime}\right)$ in Eq. (27) the disturbance $D_{\mathcal{E}}\left(\mathcal{M}^{\theta}, \sigma_{x}\right)$ is very sensitive to the input data. Unlike in 
(a)
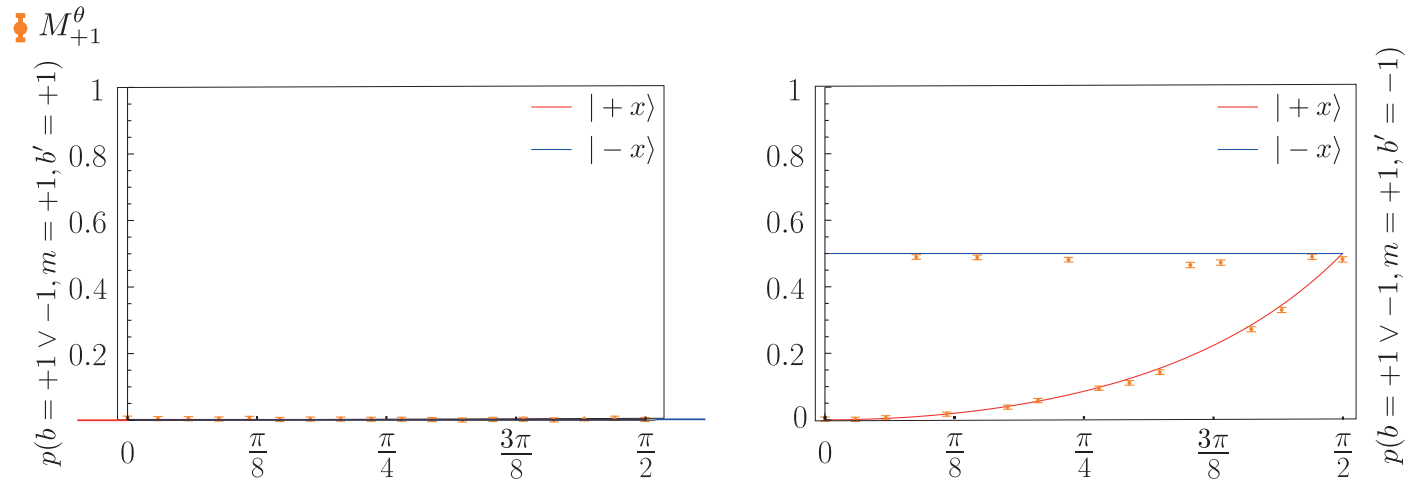

(b)
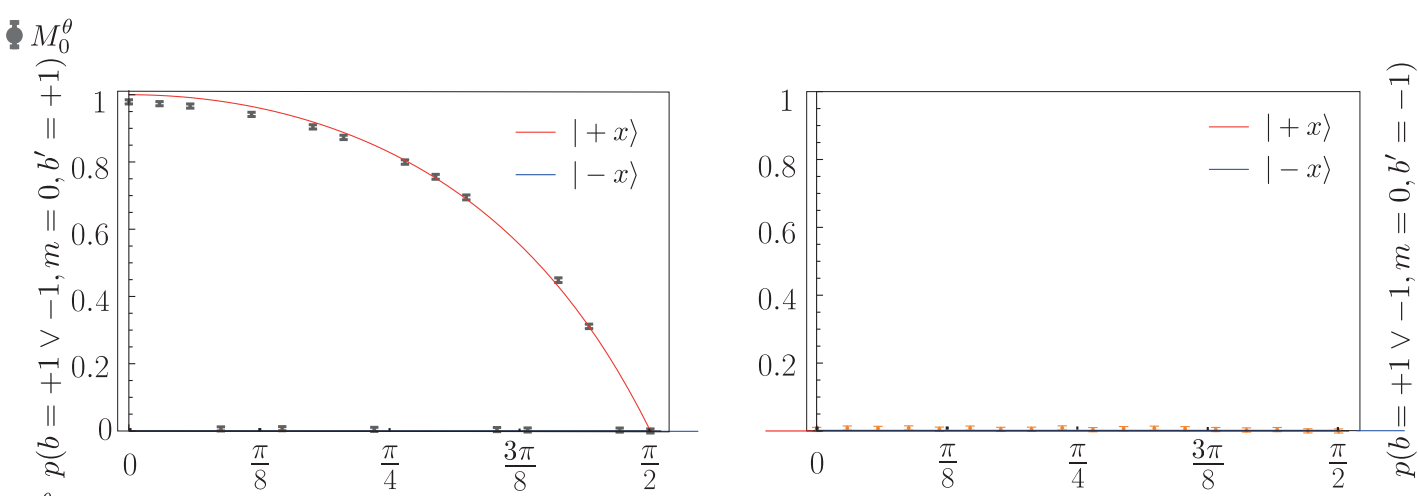

(c)
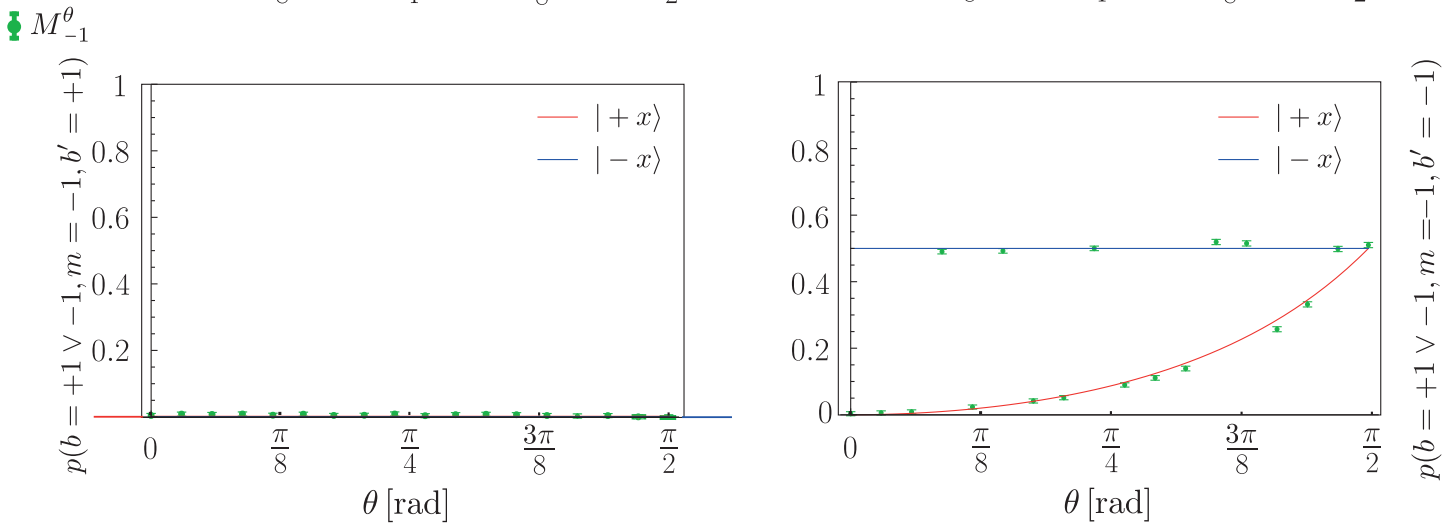

FIG. 8. Normalized data from Eq. (25), of $M_{+1}^{\theta}, M_{0}^{\theta}$, and $M_{-1}^{\theta}$, respectively, with "indefinite" input state $(|+x\rangle$ or $|-x\rangle)$ split up in the two output channels of the subsequent projective $B$ measurement with $B=\sigma_{x}$.

the case of the noise $N\left(\mathcal{M}^{\theta}, \sigma_{z}\right)$, for the disturbance certain probabilities are predicted to be zero over the entire range of $\theta$.

\section{Final results}

A parametric plot of the experimental results of the noise-disturbance measurement is given in Fig. 11, where the disturbance $D_{\mathcal{E}}\left(\mathcal{M}^{\theta}, \sigma_{x}\right)$ is plotted versus the noise $N\left(\mathcal{M}^{\theta}, \sigma_{z}\right)$. Note that the final results from Fig. 11 contain disturbance measurements of $t_{\text {meas }}=400 \mathrm{~s}$, for the first four noise-disturbance pairs (high disturbance, low noise, top left), and $t_{\text {meas }}=800 \mathrm{~s}$, for the last four noise-disturbance pairs (low disturbance, high noise, bottom right) for better statistics. Here, only noise-disturbance pairs where it is possible to decide whether projective or POVM measurements perform better (due to the size of error bars) are shown.
In addition, Fig. 11 gives an experimental comparison with the results from the projective measurements from [24], in terms of $N\left(\mathcal{M}_{\mathrm{pr}}, \sigma_{z}\right)$ versus $D_{\mathcal{E}}\left(\mathcal{M}_{\mathrm{pr}}, \sigma_{x}\right)$. Our experimental data clearly confirm that the three-outcome POVM measurement outperforms usual projective measurements, evidently reproducing the tighter bound theoretically predicted in [25].

\section{DISCUSSION AND OUTLOOK}

At this point, we want to emphasize that Fig. 10 gives an intuitive explanation why the three-outcome POVM, defined in Eq. (9), outperforms projective measurements: although there is a loss coming from the noise in the POVM (meaning higher noise values compared to the projective measurement), this loss is surpassed by the gain in the obtained disturbance (significantly lower disturbance values as for projective measurement). This behavior is a peculiarity of the applied three-outcome POVM. In general, increasing the number of 
(a) Joint probabilities:
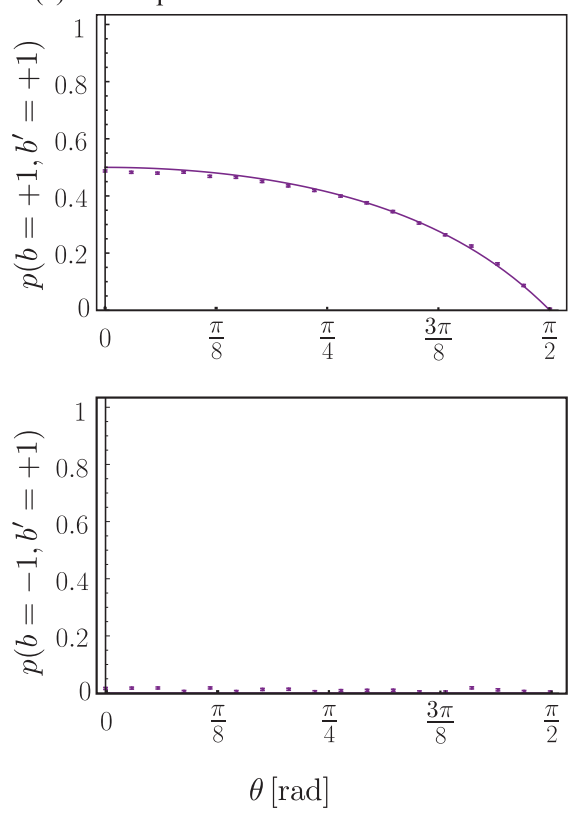

(b) Marginal probabilities:
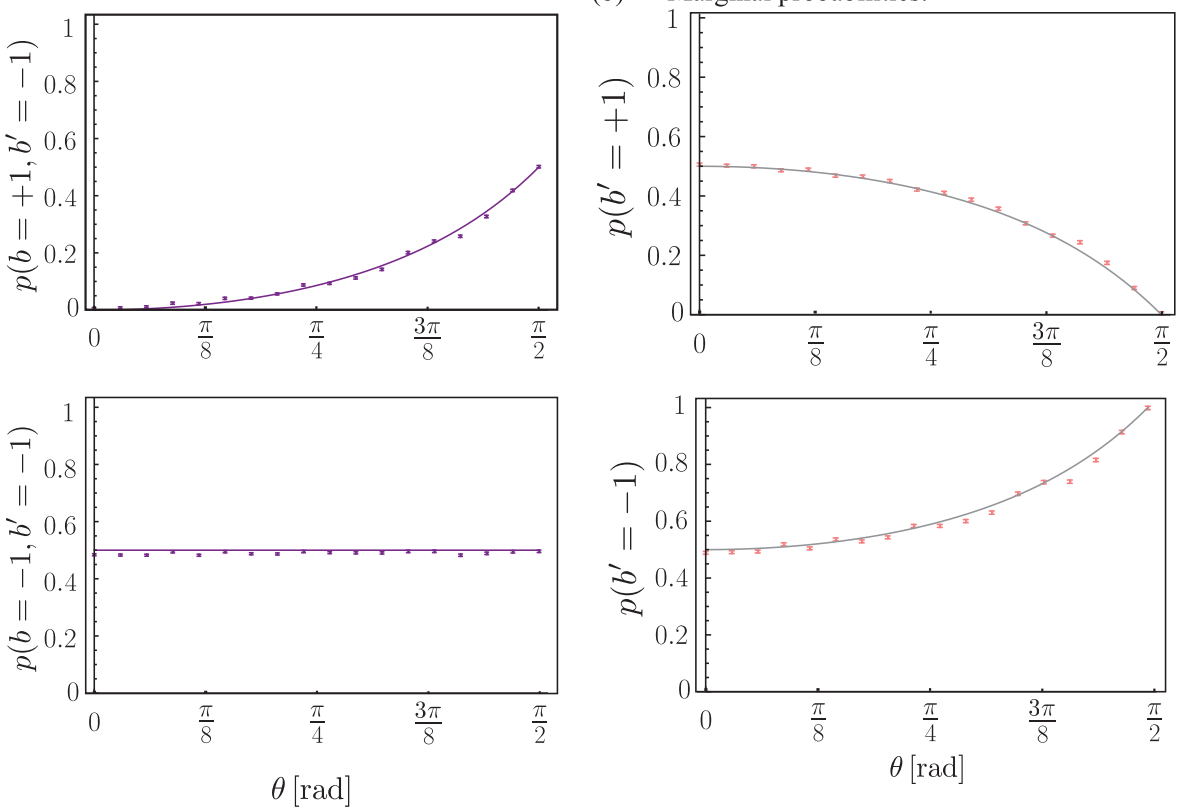

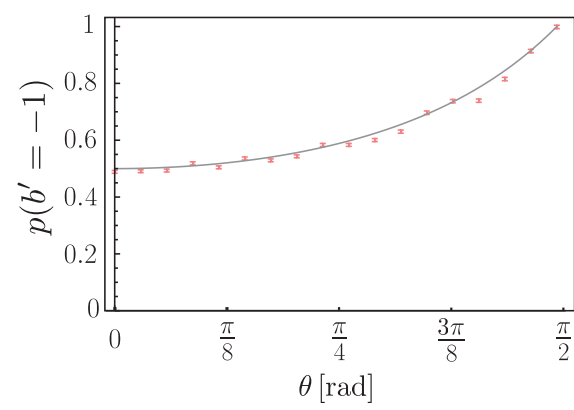

FIG. 9. (a) Joint probabilities $p\left(b, b^{\prime}\right)$ with $b= \pm 1$ and $b^{\prime}= \pm 1$, obtained via Eq. (27). (b) Marginal probabilities $p\left(b^{\prime}\right)$, by applying Eq. (29), together with theoretical predictions.

possible outcomes has a negative (increasing) effect on the noise-disturbance bound [25].

Entropic uncertainty relations have many applications in the rapidly growing field of quantum cryptography, e.g., quantum key distribution (QKD) (see for instance [32] for a recent review). The security of QKD crucially relies on Heisenberg's uncertainty principle in general, more precisely, mainly on entropic preparation uncertainty relations, since Alice (and Bob) perform measurements on one of two (randomly selected) incompatible observables (typically in $\mathbb{Z}$ and $\mathbb{X}$ basis), to generate the common secret key. For example, in Ekert's protocol [33], Alice and Bob share a maximally entangled two-qubit state in the quantum channel [12] plus a public

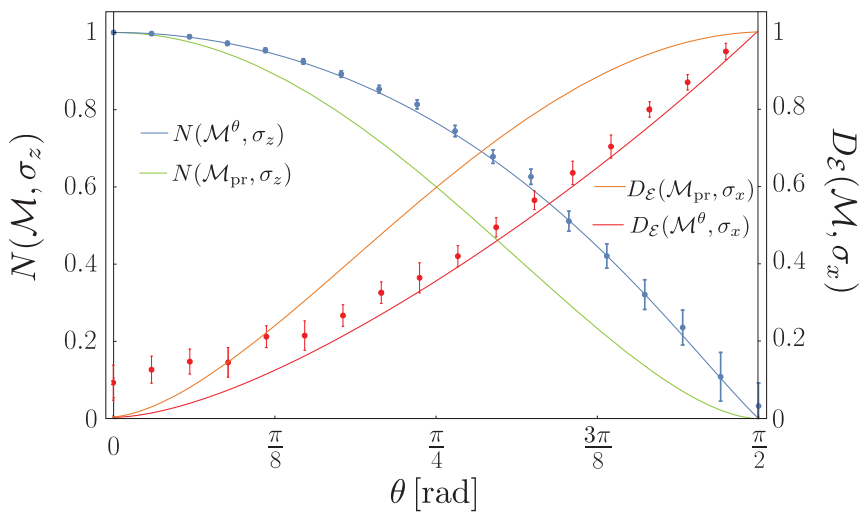

FIG. 10. Plot of noise $N\left(\mathcal{M}^{\theta}, \sigma_{z}\right)$ and disturbance $D_{\mathcal{E}}\left(\mathcal{M}^{\theta}, B\right)$ of the three-outcome POVM $M^{\theta}$ as a function of the POVM parameter $\theta$, together with the theoretical predictions (blue and red line). For comparison, theoretical prediction of $N\left(\mathcal{M}_{\mathrm{pr}}, \sigma_{z}\right)$ and $D_{\mathcal{E}}\left(\mathcal{M}_{\mathrm{pr}}, \sigma_{x}\right)$ in case of projective measurements are shown. Error bars correspond to plus or minus one standard deviation arising from the Poissonian statistics of the neutron count rate. channel; Eve (the eavesdropper) interacts via the quantum channel (e.g., by performing a measurement). Security of QKD is guaranteed if Alice and Bob have large agreement on the qubits measured in one basis, then necessarily Eve's information about the bits measured in the complementary basis must be low. When the eavesdropper interacts, for instance, when Eve is in possession of a quantum memory,

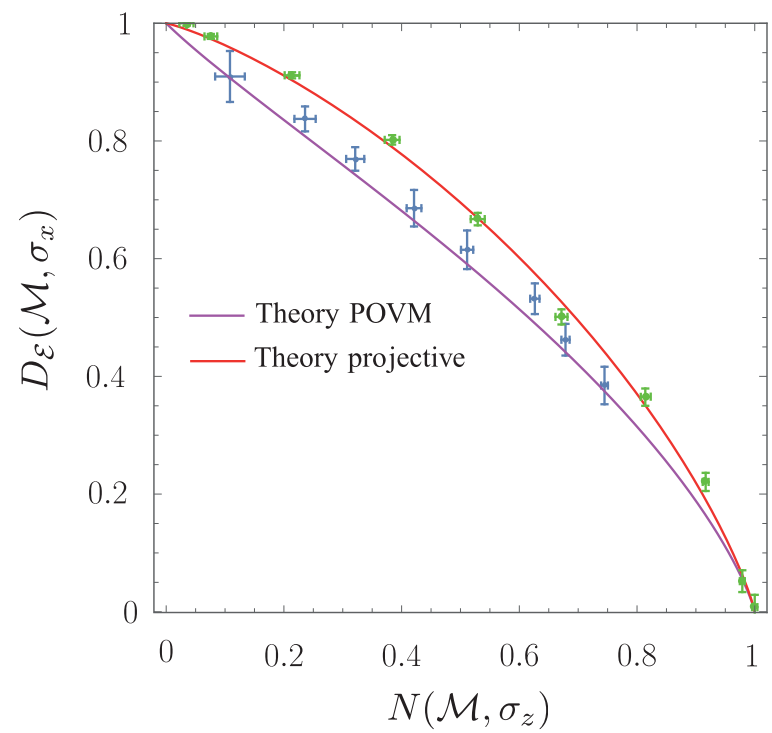

FIG. 11. Experimental comparison between noise-disturbance plot for successive projective measurements $N\left(\mathcal{M}_{\mathrm{pr}}, \sigma_{z}\right)$ vs $D_{\mathcal{E}}\left(\mathcal{M}_{\mathrm{pr}}, \sigma_{x}\right)$ (green points), taken from [24], together with theoretical predictions in red and $N\left(\mathcal{M}^{\theta}, \sigma_{z}\right)$ vs $D_{\mathcal{E}}\left(\mathcal{M}^{\theta}, \sigma_{x}\right)$ (blue points) for the three-outcome POVM $M^{\theta}$ of the measurement apparatus $\mathcal{M}^{\theta}$, with theory in purple. Error bars correspond to plus or minus one standard deviation. 
one has to assume that Alice, Bob, and Eve share a common state after the preparation. In this case, a tripartite entropic uncertainty relation in the presence of a quantum memory [12] has to be considered. Our approach presented here focuses on characterization of a single measurement and its disturbance on another, subsequent measurement in terms of entropy. It is relevant to evaluate individual measurements represented by POVMs. The tested tight measurement uncertainty relation (7) expresses the loss of correlations caused by the measurement itself.

A next experimental step will be investigation of two consecutive three-outcome POVMs. So far, only the first measurement apparatus used a POVM followed by a subsequent projective measurement. It is of interest to replace the projective measurement apparatus with a second three-outcome POVM and study the resulting disturbance on the second POVM.

\section{CONCLUSION}

We experimentally tested a tight information-theoretic measurement uncertainty relation, in terms of a proposed three-outcome POVM using neutron spin- $\frac{1}{2}$ qubits. The obtained results of the noise-disturbance tradeoff relation for three-outcome POVM outperform prior results for projective measurements, over almost the entire measured range of the tested POVM parameter $\theta$.

\section{ACKNOWLEDGMENTS}

The authors thank A. A. Abbott and C. Branciard for helpful discussions. This work was supported by the Austrian science fund (FWF) Projects No. P 30677-N36 and No. P 27666-N20.
[1] W. Heisenberg, Über den anschaulichen inhalt der quantentheoretischen kinematik und mechanik english translation: Quantum mechanical reinterpretation of kinematic and mechanical relations, Z. Phys. 43, 172 (1927).

[2] E. H. Kennard, Zur Quantenmechanik einfacher Bewegungstypen, Z. Phys. 44, 326 (1927).

[3] H. P. Robertson, The uncertainty principle, Phys. Rev. 34, 163 (1929).

[4] D. Deutsch, Uncertainty in Quantum Measurements, Phys. Rev. Lett. 50, 631 (1983).

[5] M. Ozawa, Universally valid reformulation of the Heisenberg uncertainty principle on noise and disturbance in measurement, Phys. Rev. A 67, 042105 (2003).

[6] M. Ozawa, Soundness and completeness of quantum rootmean-square errors, npj Quantum Inf. 5, 1 (2019).

[7] I. I. Hirschman, A note on entropy, Am. J. Math. 79, 152 (1957).

[8] W. Beckner, Inequalities in Fourier analysis, Ann. Math. 102, 159 (1975)

[9] I. Białynicki-Birula and J. Mycielski, Uncertainty relations for information entropy in wave mechanics, Commun. Math. Phys. 44, 129 (1975).

[10] H. Maassen and J. B. M. Uffink, Generalized Entropic Uncertainty Relations, Phys. Rev. Lett. 60, 1103 (1988).

[11] K. Kraus, Complementary observables and uncertainty relations, Phys. Rev. D 35, 3070 (1987).

[12] M. Berta, M. Christandl, R. Colbeck, J. M. Renes, and R. Renner, The uncertainty principle in the presence of quantum memory, Nat. Phys. 6, 659 (2010).

[13] P. J. Coles, J. Kaniewski, and S. Wehner, Equivalence of waveparticle duality to entropic uncertainty, Nat. Commun. 5, 5814 (2014).

[14] M. A. Nielsen and I. L. Chuang, in Quantum Computation and Quantum Information, edited by M. A. Nielsen and I. L. Chuang (Cambridge University Press, Cambridge, 2000).

[15] M. Ozawa, Uncertainty relations for noise and disturbance in generalized quantum measurements, Ann. Phys. 311, 350 (2004).

[16] R. F. Werner, The uncertainty relation for joint measurement of postion and momentum, Quantum Inf. Comput. 4, 546 (2004).
[17] F. Buscemi, M. J.W. Hall, M. Ozawa, and M. M. Wilde, Noise and Disturbance in Quantum Measurements: An InformationTheoretic Approach, Phys. Rev. Lett. 112, 050401 (2014).

[18] P. J. Coles and F. Furrer, State-dependent approach to entropic measurement-disturbance relations, Phys. Lett. A 379 , 105 (2015)

[19] K. Baek and W. Son, Entropic uncertainty relations for successive generalized measurements, Mathematics 4, 41 (2016).

[20] R. Schwonnek, D. Reeb, and R. F. Werner, Measurement uncertainty for finite quantum observables, Mathematics 4, 38 (2016).

[21] A. Barchielli, M. Gregoratti, and A. Toigo, Measurement uncertainty relations for discrete observables: Relative entropy formulation, Commun. Math. Phys. 357, 1253 (2018).

[22] E. B. Davies and J. T. Lewis, An operational approach to quantum probability, Commun. Math. Phys. 17, 239 (1970).

[23] M. Ozawa, Quantum measuring processes of continuous observables, J. Math. Phys. 25, 79 (1984).

[24] G. Sulyok, S. Sponar, B. Demirel, F. Buscemi, M. J. W. Hall, M. Ozawa, and Y. Hasegawa, Experimental Test of Entropic NoiseDisturbance Uncertainty Relations for Spin-1/2 measurements, Phys. Rev. Lett. 115, 030401 (2015).

[25] A. A. Abbott and C. Branciard, Noise and disturbance of qubit measurements: An information-theoretic characterization, Phys. Rev. A 94, 062110 (2016).

[26] B. Demirel, S. Sponar, and Y. Hasegawa, Measurements of entropic uncertainty relations in neutron optics, Appl. Sci. 10, 1087 (2020).

[27] B. Demirel, S. Sponar, A. A. Abbott, C. Branciard, and Y Hasegawa, Experimental test of an entropic measurement uncertainty relation for arbitrary qubit observables, New J. Phys. 21, 013038 (2019).

[28] G. Sulyok and S. Sponar, Heisenberg's error-disturbance uncertainty relation: Experimental study of competing approaches, Phys. Rev. A 96, 022137 (2017).

[29] B. Demirel, S. Sponar, G. Sulyok, M. Ozawa, and Y. Hasegawa, Experimental Test of Residual Error-Disturbance Uncertainty Relations for Mixed Spin-1/2 States, Phys. Rev. Lett. 117, 140402 (2016). 
[30] G. Sulyok, S. Sponar, J. Erhart, G. Badurek, M. Ozawa, and Y. Hasegawa, Violation of Heisenberg's error-disturbance uncertainty relation in neutron-spin measurements, Phys. Rev. A 88, 022110 (2013).

[31] J. Erhart, S. Sponar, G. Sulyok, G. Badurek, M. Ozawa, and Y. Hasegawa, Experimental demonstration of a universally valid error-disturbance uncertainty relation in spin-measurements, Nat. Phys. 8, 185 (2012).

[32] P. J. Coles, M. Berta, M. Tomamichel, and S. Wehner, Entropic uncertainty relations and their applications, Rev. Mod. Phys. 89, 015002 (2017).

[33] A. K. Ekert, Quantum Cryptography Based on Bell's Theorem, Phys. Rev. Lett. 67, 661 (1991). 